\title{
Enhancing the Scratch Resistance of Polycarbonate With Poly(Ethylene Oxide)-Silica Hybrid Coatings
}

\section{PAOLA FABBRI, MASSIMO MESSORI}

Dipartimento di Ingegneria dei Materiali e dell'Ambiente, Università di Modena e Reggio Emilia, Modena, Italy

Consorzio Interuniversitario Nazionale per la Scienza e la Tecnologia dei Materiali (INSTM),

Firenze, Italy

\section{MAURIZIO TOSELLI}

Consorzio Interuniversitario Nazionale per la Scienza e la Tecnologia dei Materiali (INSTM),

Firenze, Italy

Dipartimento di Chimica Applicata e Scienza dei Materiali, Università di Bologna, Bologna, Italy

\section{PAOLO VERONESI}

Dipartimento di Ingegneria dei Materiali e dell'Ambiente, Università di Modena e Reggio Emilia, Modena, Italy

\section{JOÃO ROCHA}

Department of Chemistry, CICECO, University of Aveiro, Aveiro, Portugal

\section{FRANCESCO PILATI}

Dipartimento di Ingegneria dei Materiali e dell'Ambiente, Università di Modena e Reggio Emilia, Modena, Italy

Consorzio Interuniversitario Nazionale per la Scienza e la Tecnologia dei Materiali (INSTM),

Firenze, Italy 


\section{SCRATCH-RESISTANT PEO-SiO ${ }_{2}$ COATINGS FOR POLYCARBONATES}

ABSTRACT: Scratch-resistant coatings for bisphenol-A polycarbonate sheets were obtained by the sol-gel synthesis of an organic-inorganic hybrid system based on poly(ethylene oxide) and silica. The organic-inorganic hybrids were thermally cured into hard transparent coatings by using conventional and microwave (MW) ovens. Both techniques proved to be equally efficient in promoting the system's crosslinking, as evaluated by ${ }^{29} \mathrm{Si}$ MAS-NMR. The MW-assisted curing, however, was much faster. Photoelasticity analysis showed that MW-assisted curing causes localized overheating of the samples, inducing a state of residual plane stresses that bring about dimensional instability of the coated material. Instrumented scratch tests for the coated samples revealed an increase of 1 order of magnitude in the minimal load at which a scratch track appears on the sample surface. However, the friction coefficient values for samples with thermally cured coatings were lower than those produced by MW-assisted curing. (c) 2009 Wiley Periodicals, Inc. Adv Polym Techn 27: 117-126, 2008; Published online in Wiley InterScience (www.interscience.wiley.com). DOI 10.1002/adv.20122

KEY WORDS: Coatings, Microwave curing, Organic-inorganic hybrids, Polycarbonates, Surfaces

\section{Introduction}

$\mathbf{T}$ he poor wear and scratch resistance typical of transparent polymeric materials such as polycarbonates often represents their main limiting factor in all those applications in which the contact with dust or abrasive agents cannot be avoided. Helmet visors or windowpanes are notable examples of materials for which the retention of clarity and gloss of the material over the years is mandatory for the successful performance of these devices.

For this reason, the development of permanently transparent hard coatings for plastic substrates is encouraged by commercial enterprises for the substitution of inorganic glasses with safer and lighter polymeric materials in several applications.

To this end, different approaches have been suggested for the protection of transparent plastics by means of protective coatings. Within this framework, bisphenol-A polycarbonate (PC) has received a lot of attention for its all-round good properties and, in particular, its superior toughness over other commercial glassy polymers.

At present, hard coatings on PC are mainly applied in the form of lacquers, although the lacquering techniques have some disadvantages, such as the high scrap rate for parts with a complex geometry. Physical vapor deposition (PVD) or plasmaenhanced chemical vapor deposition (PECVD) are also used for the production of coating materials with excellent abrasion resistance and thermocycling performance. ${ }^{1,2}$ In these cases, however, the resultant coatings tend to have a poor adhesion toward the substrate, mainly owing to the large difference in thermal expansion coefficients and elastic moduli between the coating and substrate materials. Other drawbacks include the risk of surface degradation of the polymer substrate. The costs related to vacuum technology are also important limitations for these processes.

The coating of plastic substrates by wet chemical processes, such as the sol-gel technique, often represents the most attractive alternative to PECVD, thanks to the extreme versatility of this approach and to the mild and inexpensive preparation conditions required. Transparent organic-inorganic hybrid coatings with covalent linkages between the organic and inorganic networks can be easily prepared by the sol-gel method, giving rise to more flexible and adherent coatings starting from metal alkoxides (typically tetraethoxysilane, TEOS). In the presence of organic oligomers or polymers, these can form a three-dimensional metal oxide network through hydrolysis and condensation reactions, which incorporates such organic components to produce organicinorganic hybrids (also known as ceramers).

These materials are phase-interconnected nanocomposites resulting from the high level of interpenetration between organic and inorganic phases and are transparent because the domain 
phase size is within the nanometer scale. Ceramers have already been recognized as interesting coating materials (a) for the improvement of barrier properties in the field of polymer-based packaging materials, ${ }^{3,4}$ (b) for the prevention of plasticizer leaching from poly(vinyl chloride) medical devices, ${ }^{5}$ and (c) for the improvement of the corrosion resistance of various metals. ${ }^{6-8}$

Furthermore, a marked improvement of both flame and scratch resistance of plastic substrates has recently been reported by using protective coatings based on ceramers prepared by the sol-gel method. $^{9-11}$ Several ultraviolet-curable hard transparent hybrid coatings on PC, based on acrylates and methacrylates as organic phase, were also successfully prepared by the sol-gel process. ${ }^{12-15}$

In this paper, poly(ethylene oxide) (PEO)-silica hybrid coatings were prepared by the sol-gel method and applied onto PC to improve its scratch resistance. Poly(ethylene oxide) was chosen as the organic part of the coatings owing to its miscibility and thermodynamic affinity with PC. ${ }^{16}$ Conventional thermal curing and microwave (MW)-assisted curing were used to obtain hard transparent coatings from the hybrid sols, and their ability to promote the hybrid system crosslinking and their effect on the final properties of the coated PC were compared.

Several advantages can be derived from the industrial application of the approach proposed in this study, such as the reduced costs related to process technology and precursor chemicals compared with the PVD or PECVD techniques. In addition, the very reduced time needed for MW-assisted hardening of the coating represents another strong point in favor of the commercial application of the system discussed.

\section{Experimental}

\section{MATERIALS}

$\alpha, \omega$-Hydroxy-terminated PEO (purchased from Fluka, Milan, Italy and with a number average molecular weight of about $1000 \mathrm{~g} \mathrm{~mol}^{-1}$ ), 3-isocyanatopropyltriethoxysilane (ICPTES, Fluka, Milan, Italy), TEOS (Aldrich, Milan, Italy), hydrochloric acid at 37\% concentration (Carlo Erba), ethanol (EtOH, Carlo Erba), and $n$-butanol $(\mathrm{BuOH}$, Carlo Erba, Milan, Italy) were obtained as high-purity reagents and used without further purification.
Sheets of PC (Makroclear $^{\mathrm{TM}}$ provided by Arla Plast, Sweden) were cut into slabs having dimensions of $5 \mathrm{~mm} \times 35 \mathrm{~mm} \times 35 \mathrm{~mm}$ and used as substrate for coating application.

\section{PREPARATION OF $\alpha, \omega$-TRIETHOXYSILANE-TERMINATED PEO}

$\alpha, \omega$-Triethoxysilane-terminated PEO (PEOSi) was prepared by the bulk reaction of PEO with ICPTES (molar ratio of 1:2) following a previously reported procedure. ${ }^{5}$ The molecular structure of the final product can be represented as follows: $(\mathrm{EtO})_{3} \mathrm{Si}-$ $\mathrm{PEO}-\mathrm{Si}(\mathrm{OEt})_{3}$.

\section{PREPARATION OF ORGANIC-INORGANIC HYBRID COATINGS}

Mixtures of TEOS and PEOSi were dissolved in $\mathrm{BuOH}$ at a concentration of $10 \%, 20 \%$, and $40 \%$ $\mathrm{wt} / \mathrm{vol}$, then water (for the hydrolysis reaction), $\mathrm{EtOH}$ (to enhance miscibility), and hydrochloric acid (as catalyst) were added in the following molar ratios, with respect to ethoxide groups belonging to either TEOS or PEOSi: EtO-: $\mathrm{H}_{2} \mathrm{O}: \mathrm{EtOH}: \mathrm{HCl}=$ 1:1:1:0.05.

A typical preparation for $\mathrm{PEOSi}-\mathrm{SiO}_{2}$ hybrids is as follows: PEOSi $(1.20 \mathrm{~g})$ and TEOS (0.80 g) were added to $8 \mathrm{~mL}$ of $\mathrm{BuOH}$ in a screw-thread glass vial and mixed until complete dissolution. Then water, $\mathrm{EtOH}$, and $\mathrm{HCl}(37 \%$ wt solution) were dropwise added under vigorous stirring at room temperature. The closed vial was placed in an air-circulating oven at $90^{\circ} \mathrm{C}$ for $45 \mathrm{~min}$ to allow a partial progress of the sol-gel reaction. After cooling and filtration (PTFE filter with a porosity of $0.45 \mu \mathrm{m})$, the clear solution was deposited onto PC slabs (previously rinsed in $\mathrm{BuOH}$ ) by spin coating (Laurell WS-400B-NPP-Lite spin coater operating at a spin rate of $1000 \mathrm{rpm}$ over $30 \mathrm{~s}$ ). Coatings were deposited at room temperature and $50 \%$ relative humidity and allowed to dry before curing.

The hybrid coatings contained a final organic-toinorganic weight ratio of 20:80, assuming the completion of the sol-gel reactions as represented in Scheme 1. These hybrids were coded $\mathrm{PEOSi}-\mathrm{SiO}_{2}$ $20 / 80$.

\section{CURING TREATMENTS}

The hybrid solutions deposited by spin coating onto PC substrates were cured into transparent, 


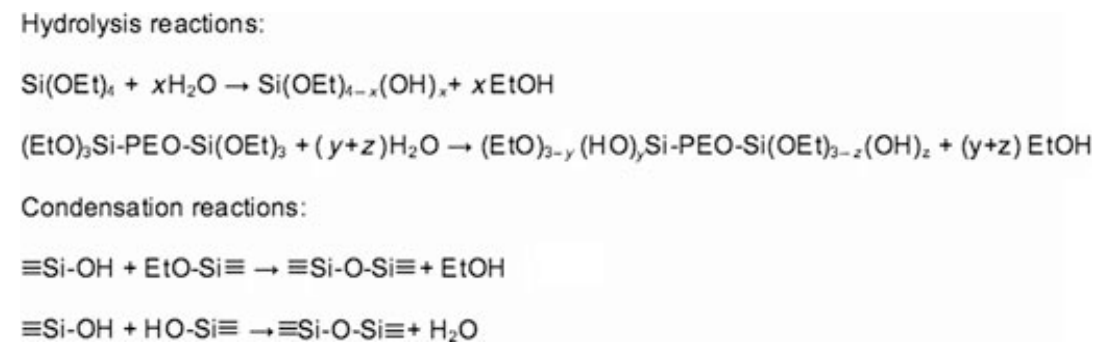

SCHEME 1. Sol-gel reactions involved in the process.

abrasion-resistant coatings by means of different postcuring treatments, namely, thermal or MWassisted curing. The efficiency of different curing techniques was further evaluated by means of nuclear magnetic resonance (NMR) spectroscopy to determine the extent of condensation reactions within the siloxane domains.

Since PC has a glass transition temperature in the region of $150^{\circ} \mathrm{C}$, thermal heating was performed over $24 \mathrm{~h}$ in an air-circulating oven (WTB Binder, Tuttlingen, Germany) at a temperature limited to $140^{\circ} \mathrm{C}$ to avoid warping and dimensional changes of the substrate. Microwave irradiation was generated by using an Alter TE10n single-mode 2.45-GHz applicator. The magnetron power was kept at $100 \mathrm{~W}$ for an irradiation time of $35 \mathrm{~s}$. The specimen was placed horizontally inside the MW applicator, with respect to the radiation source.

\section{CHARACTERIZATION}

${ }^{29}$ Si MAS-NMR spectra of the thermally and MWcured hybrids were recorded on a Bruker Avance 400 (9.4 T) DSX spectrometer operating at $79.49 \mathrm{MHz}$, with 2 - $\mu \mathrm{s}\left(\mathrm{ca} .40^{\circ}\right)$ radiofrequency pulses, 60-s recycle delay, and $5-\mathrm{kHz}$ spinning rate. Chemical shifts are quoted in parts per million (ppm) with respect to tetramethylsilane, using kaolinite as a secondary standard. Spectra were recorded on the cured bulky $\mathrm{PEOSi}-\mathrm{SiO}_{2}$ hybrids obtained either by thermal heating for $24 \mathrm{~h}$ at $140^{\circ} \mathrm{C}$ or by $\mathrm{MW}$ irradiation at $100 \mathrm{~W}$ for $35 \mathrm{~s}$. Spectra were deconvoluted with Gaussian curves of the same full-width-half maximum to calculate the degree of condensation of the hybrids as $\sum_{n} n Q_{n} / f$, where $f$ is the maximum of connectivity for a precursor, $n$ is the connectivity of the silicon site, and $Q$ represents the area of the corresponding NMR signal having the connectivity $n$.

The state of residual plane stress present in the PC slabs, before and after the coating application and curing, was evaluated by a photoelasticity analysis using a transmission photoelastic meter equipped with a white light source, determining the isochromatic lines of the first orders comparable with fringe patterns.

Coated PC slabs were fractured in liquid nitrogen and fracture surfaces of the coated specimens were gold-coated and examined by scanning electron microscopy (SEM) using a Philips XL-30 instrument.

Scratch test was carried out on a CSM MicroCombi tester. In this test, a controlled scratch on the coating surface is made with a diamond tip (Rockwell $C$ diamond scratch indenter with tip radius of $800 \mu \mathrm{m})$ on the sample. The tip is drawn across the coated surface under progressive load (from $100 \mathrm{mN}$ to $20 \mathrm{~N}$ ) at a load rate of $4.98 \mathrm{~N} \mathrm{~min}^{-1}$ over a scratch length of $1 \mathrm{~mm}$ (see Fig. 1).

The instrument bears an integrated optical microscope, an acoustic emission detection system, and a device measuring the tangential frictional force (in the scratch direction), which is related to the friction coefficient. The critical load for a coating-substrate system was determined by optical methods. Three scratches were carried out in different zones for each specimen and average values of the load at which the scratch track appears $\left(L_{\mathrm{c} 1}\right)$, as well as of the load at which the detachment of the coating occurs (critical load, $L_{\mathrm{c} 2}$ ), were determined for each analysis. Furthermore, the tangential frictional force was determined for the evaluation of the friction coefficient between the tip and the coating surface.

\section{Results and Discussion}

The performance of a coating layer to protect the substrate from scratch damages strongly depends on the intrinsic properties of the coating material, such as its chemical composition, and coating film 


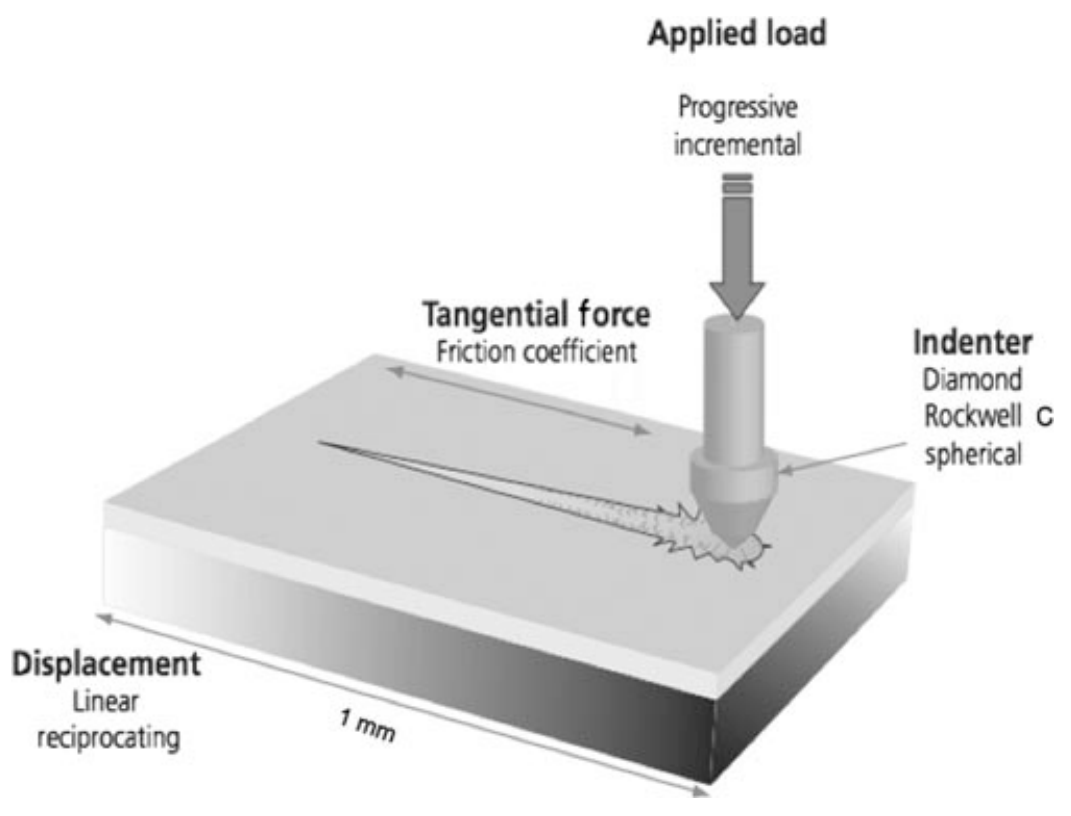

FIGURE 1. Scheme of the scratch test performed on the samples.

properties such as thickness, hardness, and adhesion to the substrate. These latter features, in the case of sol-gel-derived organic-inorganic hybrids, can be tuned to a large extent by a judicious choice of chemical precursors in the recipe, the amounts used, and the way in which they are cured into a permanent hard coating.

In the present work, all the coatings were prepared starting from PEO silane-functionalized polymer chains (PEOSi) and TEOS and applied onto PC slabs by spin coating to obtain smooth coatings with uniform thickness. Irrespective of the curing treatment adopted, all samples were highly transparent and homogeneous. This can be taken as evidence of the presence of nanosized domains in the coatings. ${ }^{17}$

This confirms that both thermal curing and $\mathrm{MW}$-assisted curing are effective in promoting the crosslinking of the hybrid system, but there are no indications of the network density and degree of connectivity of the constituent domains.

The SEM analysis was used to measure the coating thickness, which affects the antiscratch properties of the coatings. The thickness of the coatings was found to be dependent on the concentration of the starting sol-gel solution (the more highly concentrated solutions had higher viscosity and produced thicker coatings), although the thickness was found to be almost independent of the type of curing treatment used. The dependence of the final thickness of the coatings on the concentration of the precursor sol-gel solution is reported in Fig. 2. This shows that coatings with thickness ranging from 85 to $325 \mathrm{~nm}$ were obtained.

\section{${ }^{29}$ SI MAS-NMR}

Several spectroscopic investigations on organicinorganic hybrid materials obtained by the sol-gel process have been devoted to the study of the

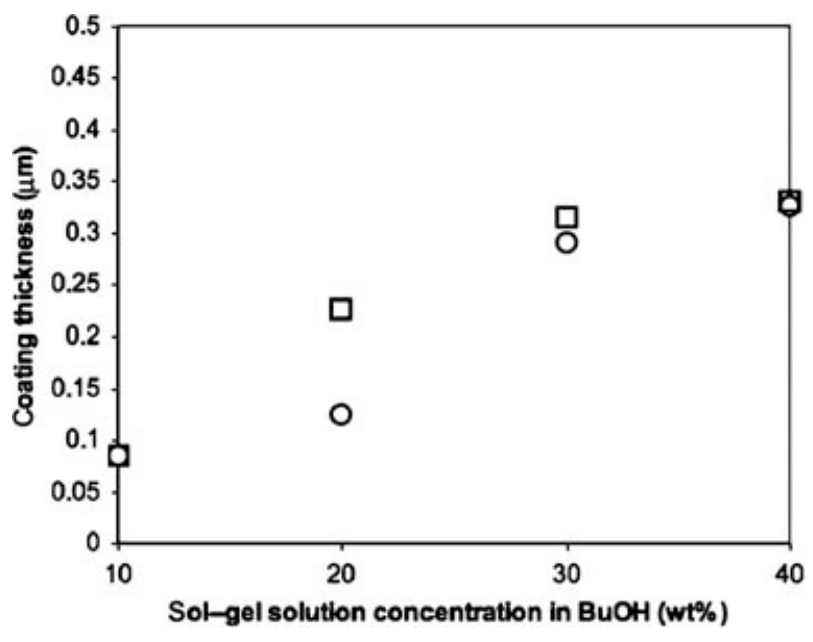

FIGURE 2. Variation of coating thickness as a function of the sol concentration and the type of curing treatment (squares are for microwave-assisted curing and circles for thermal curing). 


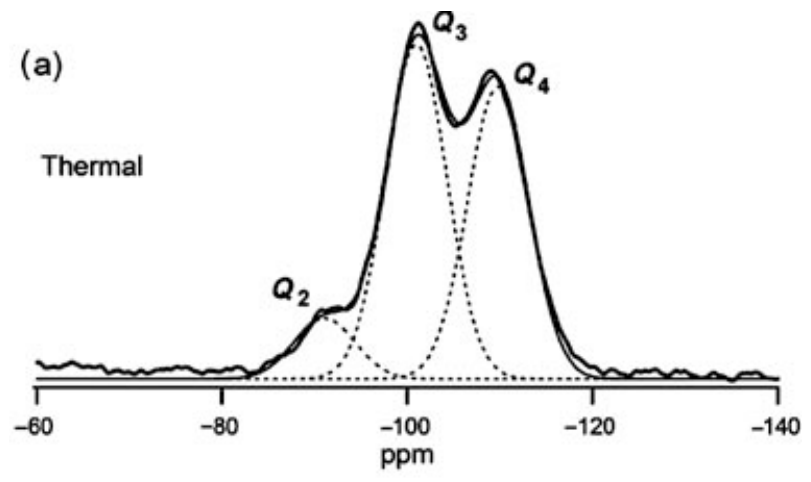

(b)

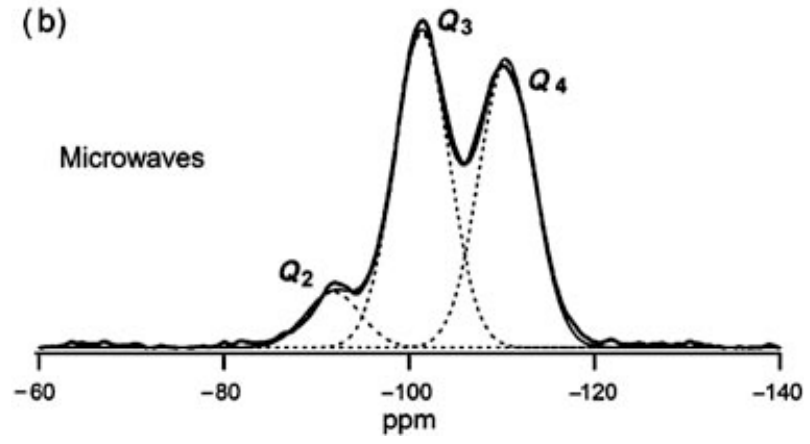

FIGURE 3. ${ }^{29}$ Si MAS-NMR spectra of thermally (a) and microwave (b) cured PEOSi-SiO 2 hybrids.

structure of the network formed as a consequence of the polycondensation reactions taking place during the curing process. ${ }^{18,19}$ In particular, ${ }^{29} \mathrm{Si}$ MAS-NMR analysis makes it possible to obtain a precise and quantitative assignment of the peaks to the tetrafunctional $(Q)$ units, more precisely to $Q_{2}, Q_{3}$, and $Q_{4}$ units, where the usual notation $Q_{n}$ for tetrafunctional $\mathrm{SiO}_{4}$ units is used, with $n$ denoting the number of bridging oxygen atoms.

In the present work, the main aim of ${ }^{29} \mathrm{Si}$ MASNMR investigation was to find a possible correlation between the performance of the hybrid material as scratch-resistant coating for PC substrates and the degree of condensation of its inorganic phase deriving from TEOS. While it is well known that in general the abrasion resistance of a material can be correlated to its surface hardness, ${ }^{20}$ for organic-inorganic hybrid materials, the latter can be further linked to the crosslinking density reached by means of postcuring treatments. ${ }^{15,21,22}$ For this reason, ${ }^{29}$ Si MAS-NMR represents a useful tool for a direct comparison of the effectiveness of thermal and MW-assisted curing of organic-inorganic hybrid materials.

Figure 3 shows the peak fitting of the $Q$ set of peaks of ${ }^{29} \mathrm{Si}$ MAS-NMR spectra, in the spectral region between -140 and $-60 \mathrm{ppm}$, for the PEOSi$\mathrm{SiO}_{2}(20 / 80 \mathrm{wt} / \mathrm{wt})$ dried sols subjected to either $\mathrm{MW}$-assisted or thermal curing.

The species identified in each system are listed in Table I, along with their chemical shifts and peak areas, which were used to calculate the $Q_{4}: Q_{2}$ and $Q_{3}: Q_{2}$ ratios and the TEOS condensation degree (CD) by the following formula:

$$
\operatorname{TEOSCD}(\%)=\frac{1}{4}\left(\frac{4 Q_{4}+3 Q_{3}+2 Q_{2}}{Q_{4}+Q_{3}+Q_{2}}\right) \times 100
$$

The data show that CD of the TEOS phase in the hybrid system as a consequence of different curing treatments reaches almost the same value, and the network appears highly crosslinked in both cases. Slight differences are noted in the $Q_{4}: Q_{2}$ ratio, which is slightly higher for the MW-cured sample.

This first result is a tool of primary importance for the comparison of the effectiveness of thermal and MW-assisted curing for hybrid systems, as it is evident that further differences that can be observed in terms of material hardness and scratch resistance cannot be attributed to a different crosslinking degree of the hybrid network inside the coating layer. It must be remarked, however, that the same degree

TABLE I

\begin{tabular}{lcccccc} 
Results of ${ }^{29}$ Si MAS-NMR & \multicolumn{1}{l}{} \\
Sample & Species & $\begin{array}{c}\text { Chemical } \\
\text { Shifts }( \pm 0.1 \mathrm{ppm})\end{array}$ & $\begin{array}{c}\text { Peak Area } \\
\text { (a.u.) }\end{array}$ & $\begin{array}{c}Q_{4}: Q_{2} \\
\text { Ratio }\end{array}$ & $\begin{array}{c}Q_{3}: Q_{2} \\
\text { Ratio }\end{array}$ & $\begin{array}{c}\text { Tetraethoxysilane } \\
\text { Condensation Degree (\%) }\end{array}$ \\
\hline Microwave curing & $Q_{4}$ & -110.5 & 3.63 & 5.2 & 5.7 & 83.8 \\
& $Q_{3}$ & -101.3 & 3.99 & & & \\
\multirow{4}{*}{ Thermal curing } & $Q_{2}$ & -91.8 & 0.70 & & & \\
& $Q_{4}$ & -109.8 & 3.54 & 4.8 & 5.5 & \\
& $Q_{3}$ & -100.9 & 4.05 & & & \\
& $Q_{2}$ & -91.1 & 0.73 & & & \\
\end{tabular}


of crosslinking is obtained by MW-assisted curing in much shorter time with respect to the thermal treatment, and this represents a significant advantage in industrial applications.

\section{PHOTOELASTICITY ANALYSIS}

The choice of the most appropriate curing treatment for a surface-coated product is not only a matter of industrial efficiency or convenience but also concerns with problems of dimensional stability and properties retention during the entire life cycle of the product.

Photoelasticity analysis is an effective tool to detect and estimate the magnitude of residual ("frozen") stresses set up during curing and subsequent fast cooling of the coated sample.

However, owing to the small thickness of the coating $(85-325 \mathrm{~nm})$ relative to that of the substrate $(0.4 \mathrm{~cm})$, no information can be drawn by this technique regarding the residual stress state of the actual coating layer. Pictures of pristine PC slab and PC coated with thermal- or MW-cured hybrids are reported in Fig. 4. The presence of notches on the backside of the PC slabs is due to a machining operation done on the substrate before the coating application to localize the subsequent fracture of the specimen in liquid nitrogen for SEM analysis.

The characteristic color of the isochromatic fringes can be considered as a direct measure of the deviatoric (tensional) state of stresses in the sample. The color palette (not detectable in the actual prints) varies from pale yellow-dull red (fringe order 0-0.9) to rose red (fringe order 2) to red-green (typical of fringe order 3), indicating the increase in tensional state of residual stresses.

The uncoated PC showed a quite uniform residual stress state, which obviously increases in the proximity of the notches. The PC slabs coated with the thermally cured hybrid showed a slight and uniform residual stress state, with only a slight increase in the proximity of the notches. On the contrary, the MWassisted curing produces a highly inhomogeneous residual stress state owing to a nonuniformity of the temperature resulting from MW irradiation and the subsequent fast cooling.

Similar results were obtained also for different thickness and different organic-inorganic weight ratios of the coating (results not reported in this paper), indicating that the curing treatment was the main factor responsible for the residual stresses.

It can be presumed that thermal curing with a prolonged heating period at $140^{\circ} \mathrm{C}(24 \mathrm{~h})$ causes the relaxation of frozen stresses already present, arising during the fabrication process (sheet extrusion).

Photoelasticity analysis showed that thermal curing is more suitable than the MW-assisted curing for the preparation of parts with virtually no residual stresses.

\section{SCRATCH RESISTANCE}

In the field of protective and scratch-resistant coatings, scratch tests are widely used to evaluate the mechanical strength of the coating and its adhesion to the substrate. The test used in this work induces failure in the coated sample in a rather complex way, depending not only on the intrinsic interactions between the two components of the system but also on the physical interactions between the indenter and the surface of the sample. The strength of the interfacial adhesion between the coating layer and the substrate, and of the coating layer itself, is characterized by a critical load $\left(L_{\mathrm{c}}\right)$, which is the minimum load at which damage takes place through loss of adhesion to the substrate.

The critical load depends not only on the coating adhesion to the substrate but also on several other parameters, some of which are directly related to the test itself (the intrinsic parameters) whereas others are related to the coating-substrate combination (the extrinsic parameters). ${ }^{23}$
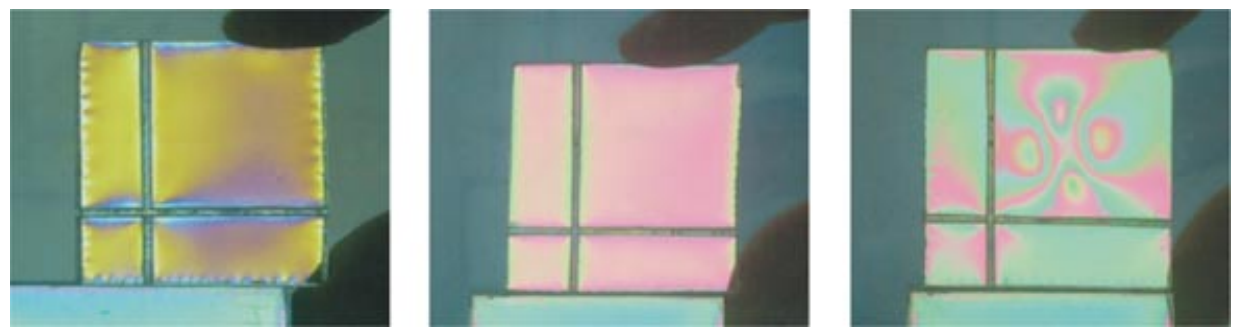

FIGURE 4. Photoelasticity analysis of uncoated and uncured bisphenol-A polycarbonate (PC) (left); PC coated with $\mathrm{PEOSi}-\mathrm{SiO}_{2}$ 20/80, thermal curing (center); and PC coated with $\mathrm{PEOSi-SiO} 2$ 20/80, microwave curing (right). 


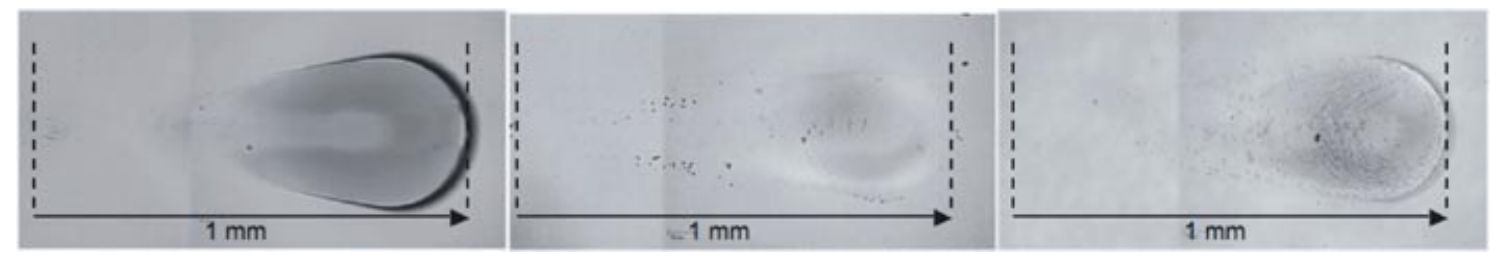

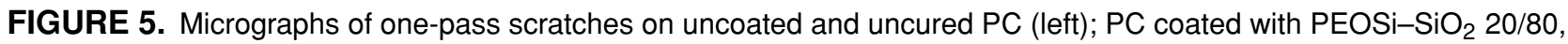
thermal curing (center); and $\mathrm{PC}$ coated with $\mathrm{PEOSi}-\mathrm{SiO}_{2} 20 / 80$, microwave curing (right). The stylus sliding direction and the scratch length are indicated by the arrow.

In the present work, both the critical load (here indicated as $L_{\mathrm{c} 2}$ ) and the lowest load at which a scratch track on the coating first appears $\left(L_{\mathrm{c} 1}\right)$ were determined. The latter parameter is usually not reported in the literature, but it can be particularly indicative of the scratch resistance of transparent coatingsubstrate assemblies taking into account that their application in optical components can be strongly limited by the formation of scratches, creating local light scattering spots.

Typical micrographs of PC slabs of both uncoated and coated samples after scratch tests are shown in Fig. 5.

The recordings in these pictures gave an $L_{\mathrm{c} 1}$ value of $0.15 \mathrm{~N}$ for uncoated PC, whereas the values for coated samples were respectively $3.41 \mathrm{~N}$ for thermally cured samples and $2.36 \mathrm{~N}$ for those cured by MW irradiation.

The same procedure was applied on at least three single-pass scratches for each sample, and the averaged results are reported in Table II. It is important to note that all the coated samples exhibited a large increase in the minimum load at which the scratch track first appears on the surface $\left(L_{\mathrm{c} 1}\right)$, relative to uncoated PC, which is indicative of the suitability of these coatings for the protection of delicate transparent optical devices, as in the case of plastic spectacle lenses.

In particular, $L_{c 1}$ has increased from the reference value of $0.12 \mathrm{~N}$ (typical of uncoated PC) to the max- imum value of $3.65 \mathrm{~N}$ registered for thermally cured PC slabs with a $0.125-\mu \mathrm{m}$ thick coating.

Another point to bear in mind is that the data reported in Table II show that the properties of the coatings deteriorate above a certain thickness, that is, both minimum load at which the first crack appears $\left(L_{\mathrm{c} 1}\right)$ and the critical load $\left(L_{\mathrm{c} 2}\right)$ decrease. The coatings show the highest scratch resistance for thicknesses in the range of $0.10-0.25 \mu \mathrm{m}$ for both thermal- and MW-cured systems. This trend confirms what other authors have reported on the relationship between scratch resistance and coating thickness. ${ }^{24}$

The initial increase of both $L_{\mathrm{c} 1}$ and $L_{\mathrm{c} 2}$ with coating thickness can be explained by taking into account the fact that the extent of deformation caused by the scratch test is related to both substrate hardness and coating thickness. ${ }^{23}$ It can also be assumed that above a certain critical coating thickness, the presence of residual stresses in the coating layer can contribute to a decrease in the stress state for the formation and propagation of cracks in the coating layer and at the interface, thereby causing concomitant decrease in the critical load values recorded by the scratch test.

\section{FRICTION FORCE AND FRICTION COEFFICIENT}

The critical load can also be determined by measuring the load at which a sudden change in

TABLE II

Results of Scratch Test ${ }^{a}$

\begin{tabular}{|c|c|c|c|c|c|}
\hline \multicolumn{3}{|c|}{ Thermal Curing } & \multicolumn{3}{|c|}{ Microwave-Assisted Curing } \\
\hline Coating Thickness ( $\mu \mathrm{m})$ & $L_{\mathrm{c} 1}(\mathrm{~N})$ & $L_{\mathrm{c} 2}(\mathrm{~N})$ & Coating Thickness $(\mu \mathrm{m})$ & $L_{\mathrm{c} 1}(\mathrm{~N})$ & $L_{\mathrm{c} 2}(\mathrm{~N})$ \\
\hline $0.085 \times 0.015$ & $3.40 \times 0.12$ & $13.32 \times 1.95$ & $0.085 \times 0.005$ & $0.86 \times 0.41$ & $14.02 \times 3.02$ \\
\hline $0.125 \times 0.025$ & $3.65 \times 0.48$ & $15.52 \times 0.10$ & $0.225 \times 0.025$ & $3.00 \times 1.50$ & $10.99 \times 4.17$ \\
\hline $0.290 \times 0.020$ & $2.28 \times 0.53$ & $13.25 \times 0.20$ & $0.315 \times 0.015$ & $1.67 \times 0.87$ & $6.52 \times 3.84$ \\
\hline $0.325 \times 0.025$ & $2.31 \times 1.26$ & $15.36 \times 0.51$ & $0.330 \times 0.020$ & $0.57 \times 0.35$ & $3.47 \times 0.99$ \\
\hline
\end{tabular}

${ }^{a} L_{c 1}=0.12 \mathrm{~N}$ for uncoated bisphenol-A polycarbonate slab. 


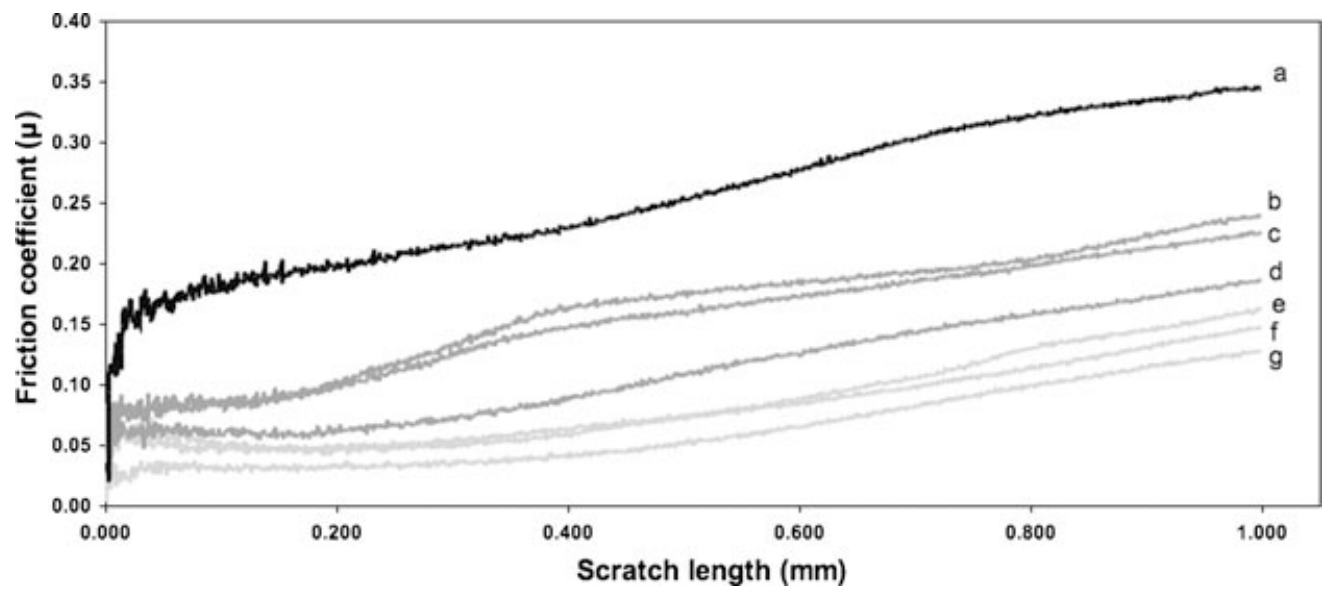

FIGURE 6. Friction coefficient between the indenter and the coating surface of bisphenol-A polycarbonate slabs uncoated (a), coated with PEOSi-SiO 2 20/80 microwave-cured (coating thickness: b, $0.225 \mu \mathrm{m} ; \mathrm{c}, 0.315 \mu \mathrm{m}$; and d, $0.085 \mu \mathrm{m}$ ) and thermally cured (coating thickness: e, $0.290 \mu \mathrm{m} ; \mathrm{f}, 0.085 \mu \mathrm{m}$; and g, $0.125 \mu \mathrm{m}$ ).

frictional force occurs. ${ }^{25}$ For the present systems, the plot of the frictional force versus load (or scratch length) did not provide significant features even for those samples in which a coating detachment was well evident from optical analysis.

Note that the frictional force recorded in these tests can be used to calculate the friction coefficient between the indenter and the coating surface.

In general, the critical load and the tangential force depend on the following parameters: substrate hardness, coating thickness, surface roughness, along with the friction coefficient between the indenter and the coating surface. ${ }^{23}$ In the specific case of nanostrustured organic-inorganic hybrids, the influence of surface roughness can be neglected because very smooth surfaces are commonly obtained by the hybrid sol-gel process (average roughness of the order of a few nanometers). Figure 6 reports the variation of the friction coefficient determined for PC slabs uncoated and coated with respect to the scratch length (which is linearly related to the applied normal load).

The uncoated PC slab exhibits a friction coefficient of 0.34 at the end of the scratch, whereas all the coated samples show a significantly lower friction coefficient, with values ranging from 0.24 for the MW-cured coating with thickness of $0.225 \mu \mathrm{m}$ (Fig. 6, curve b) to 0.13 for the thermally cured coatings with thickness of $0.125 \mu \mathrm{m}$ (Fig. 6, curve g). A clear correlation between friction coefficient and coating thickness was not evident; nevertheless, it is interesting to note that the PC slabs coated with thermally cured coatings (curves e, f, and g) were characterized by friction coefficient values lower than those of the MW-cured coatings (curves b, c, and d). This can be considered as an indirect indication of the fact that the homogeneous thermal curing resulted in a smoother coating surface with respect to the MW-assisted curing, which is in accordance with the results obtained by photoelasticity analysis. Moreover, the higher friction coefficient of MWcured samples could also indicate the presence of a plasticized layer caused by the possible penetration of $\mathrm{BuOH}$ into the substrate causing plasticization, facilitated by the higher localized temperatures produced during curing, as evidenced by the photoelasticity analysis.

\section{Conclusions}

The characteristics of PEO-silica hybrid coatings for scratch protection of PC have been investigated. Curing by thermal heating or MW irradiation of the hybrids has been evaluated. ${ }^{29}$ Si MAS-NMR analysis has shown that both treatment options were effective in promoting the crosslinking of the hybrid network. However, the same degree of curing was reached in $24 \mathrm{~h}$ at $140^{\circ} \mathrm{C}$ and only $35 \mathrm{~s}$ when irradiated at $100 \mathrm{~W}$.

The scratch resistance of coated PC greatly improved for both thermal-cured and MW-irradiated coatings. However, the former gave higher critical load values in the scratch tests.

The PC samples with thermally cured coatings exhibited lower friction coefficient values than those cured by MW irradiation. 


\section{Acknowledgments}

The authors thank Prof. Dr. Leno Mascia (Department of Materials, Loughborough University, United Kingdom) for the fruitful discussion of the results and Ms Elisa Ferrari for her support to experimental work.

\section{References}

1. Katsamberis, D.; Browall, K.; Iacovangelo, C.; Neumann, M.; Morgner, H. Prog Org Coat 1998, 34, 130-134.

2. Zajíckova, L.; Bursíkova, V.; Janca, J. Vacuum 1998, 50(1), 1921

3. Haas, K.-H.; Amberg-Schwab, S.; Ballweg, T.; Adv Sci Technol 2003, 31, 581-592.

4. Utz, H.; Amberg-Schwab, S.; Schottner, G. US Patent 6,503,634, 2003

5. Messori, M.; Toselli, M.; Pilati, F.; Fabbri, E.; Fabbri, P.; Pasquali, L.; Nannarone, S. Polymer 2004, 45, 805-813.

6. Chou, T. P.; Chandrasekaran, C.; Cao, G. Z. J Sol-Gel Sci Technol 2003, 26, 321-327.

7. Bescherand, E.; Mackenzie, J. D. J Sol-Gel Sci Technol 2003, 26, 1223-1226.

8. Mascia, L.; Prezzi, L; Wilcox, G. D.; Lavorgna, M. Prog Org Coat 2006, 56, 13-22.
9. Messori, M.; Toselli, M.; Pilati, F.; Fabbri, E.; Fabbri, P.; Busoli, S. Surf Coat Int Pt B-C 2003, 86-B3, 169-246.

10. Messori, M.; Toselli, M.; Pilati, F.; Fabbri, E.; Fabbri, P.; Busoli, S.; Pasquali, L.; Nannarone, S. Polymer 2003, 44, 4463-4470.

11. Toselli, M.; Marini, M.; Fabbri, P.; Messori, M.; Pilati F. J SolGel Sci Technol 2007, 43, 73-83.

12. Gilberts, J.; Tinnemans, A. H. A.; Hogerheide, M. P.; Koster, T. P. M. J Sol-Gel Sci Technol 1998, 11, 153-159.

13. Sepur, S.; Kunze, N.; Werner, B.; Schmidt, H. Thin Solid Films 1999, 351, 216-219.

14. Wouters, M. E. L.; Wolfs, D. P.; Van der Linde, M. C.; Hovens, J. H. P.; Tinnemans, A. H. A. Prog Org Coat 2004, 51, 312-320.

15. Martínez Urreaga, J.; Matías, M. C.; Lorenzo, V.; De la Orden, M. U. Mater Lett 2000, 45, 293-297.

16. Li, C.; Kong, Q.; Fan, Q.; Xia, Y. Mater Lett 2005, 59, 773-778.

17. Wojcik, A.; Klein, L. C. Appl Organomet Chem 1997, 11, 129135.

18. Sassi, Z.; Bureau, J. C.; Bakkali, A. Vib Spectrosc 2002, 28, 299-318.

19. Feuillade, M.; Croutxé-Batghorn, C.; Carré, C. Prog Solid State Chem 2006, 34, 87-94.

20. Stachowiak, G. W. Mater Forum 1998, 22, 63-77.

21. Mackenzie, J. D.; Bescher, E. P. J Sol-Gel Sci Technol 2000, 19, 23-29.

22. Li, C.; Jordens, K.; Wilkes, G. L. Wear 2000, 242, 152-159.

23. Steinmann, P. A.; Tardy, Y.; Hintermann, H. E. Thin Solid Films 1987, 154, 333-349.

24. Malzbender, J.; De With, G. Surf Coat Technol 2001, 135, 202207.

25. Bull, S. J.; Berasetegui, E. G. Tribol Int 2006, 39, 99-114. 